\title{
Student chapters: estructuras de inserción empresarial en la formación de grado
}

Mariano González Dobra', Carolina Cuevas', Daniela Funes ${ }^{1,2}$, Francisco Cellone ${ }^{1,2}$, Manuel López $^{1,2}$, Marina Coronel ${ }^{1,2}$

${ }^{1} F a c u l t a d$ de Ciencias Naturales y Museo Universidad Nacional de La Plata ${ }^{2}$ Centro de Investigaciones Geológicas (UNLP-CONICET) mariano.gonzalez04@hotmail.com
Resumen: Se analiza el funcionamiento de las estructuras conocidas como Student Chapters,enparticularenlaFacultaddeCiencias Naturales y Museo de la Universidad Nacional de La Plata, y su influencia en la formación de futuros profesionales de geología. Estos capítulos son el brazo educativo de distintas asociaciones profesionales norteamericanas, auspiciadas por empresas multinacionales. Poseen una estructura piramidal que involucra a los distintos claustros universitarios y tienen como función brindar a los estudiantes una formación complementariaa los contenidos de grado en temáticas específicas, con especial énfasis en el vínculo estudiante-empresa. Las acciones que realizan se desarrollan en paralelo a las actividades de grado, sin ninguna instancia de control de la universidad. Estas organizaciones actúan bajo lógicas mercantilistas, naturalizando el vínculo entre la universidad y las empresas multinacionales. Modifican además la cultura académica generando un sentido de pertenencia hacia las empresas en detrimento de la pertenencia a la institución pública.

Palabras claves: Capítulos estudiantiles, Universidad Nacional de La Plata, cultura académica, Asociaciones profesionales americanas

\section{Student chapters: business insertion structures in the formation of degree}

Abstract: We analyze the educational structures known as Student Chapters (SC), particularly at the Facultad de Ciencias Naturales y Museo of the Universidad Nacional de La Plata, and their influence on the academic training of future geologists. SC represents the academic arms of various American professional associations, sponsored by numerous multinational companies. They have a pyramid structure and are aimed to provide additional training to students on specific subjects, with special emphasis on the student-company relationship. These activities are paralell to the clases, have no university control.

The ultimate goal is to train students next to graduate under a mercantilist logic, naturalizing the link between universities and multinational companies. Moreover, these structures create a sense of belonging which in many cases, is opposed to the sense of belonging to a public institution.

Key words: Student Chapters, Universidad Nacional de La Plata, academic training, American professional associations 


\section{Introducción}

A comienzos del siglo XXI comenzaron a funcionar en las Universidades de Argentina organizaciones conocidas como Capítulos Estudiantiles o Student Chapters (SC). Los SC se definen a sí mismos como programas co-educacionales que nuclean estudiantes y graduados/as, cuyo objetivo es "proporcionar a los estudiantes la oportunidad de desarrollar habilidades de liderazgo y servir como punto focal para el desarrollo de un sentimiento de profesionalismo a través del vínculo con profesionales de la industria"'. Los SC se originan y dependen de asociaciones científico-profesionales de origen estadounidense y se encuentran presentes en todo el mundo. Actualmente, en la Facultad de Ciencias Naturales y Museo (FCNyM) de la Universidad Nacional de La Plata (UNLP) existen dos SC dependientes de la American Association of Petroleum Geologists (AAPG) y la Society of Economic Geologists (SEG). El objetivo de este trabajo es describir el funcionamiento de los SC en general y analizar los mismos en el marco de la FCNyM considerando el vínculo entre la universidad y las empresas privadas: ¿qué generan estas organizaciones en el ámbito académico? ¿La formación que brindan es la que desea la Universidad pública? ¿Cómo influyen en la cultura académica de la institución²?

\section{Metodología}

En una primera instancia, se llevó a cabo una pormenorizada investigación bibliográfica con el objetivo de caracterizar de manera detallada los SC a nivel global y dar un marco teórico adecuado a la problemática. En base a esto, se realizaron entrevistas semiestructuradas a referentes de los SC que actúan en FCNyM y a las autoridades de dicha Facultad. Por otro lado, fueron encuestados 143 estudiantes de la carrera de Geología de tercer, cuarto y quinto año, para obtener información sobre el grado de conocimiento de los SC y su participación en los mismos. Sobre las/los participantes activos, se realizó una segunda encuesta con preguntas orientadas a profundizar sobre la dinámica de funcionamiento de estas estructuras.

Hemos optado por mantener en el anonimato los nombres de las personas entrevistadas, ya que dichas entrevistas fueron desarrolladas en el marco de la realización de un trabajo monográfico para un seminario de posgrado.

\section{Caracterización de los SC}

Los SC son el brazo educativo de asociaciones y sociedades académico-profesionales de Estados Unidos vinculadas a temáticas estratégicas de desarrollo científico y tecnológico. Del abanico de te-

\footnotetext{
${ }^{1}$ Fragmento extraído y traducido de la página web de la AAPG: www.aapg.org/global/latinamerica/students (18/06/18) 2 Según Naidorf (2005) se entiende por cultura académica "el universo de pensamiento que, aunque múltiple y diverso, caracteriza en algún sentido las maneras de ser y hacer de los científicos argentinos de la Universidad pública. La cultura académica de la Universidad está conformada por los discursos, representaciones, motivaciones, normas éticas, concepciones, visiones y prácticas institucionales de los actores universitarios acerca de los objetivos de las tareas de docencia, investigación, extensión y transferencia, que condicionan sustancialmente las maneras de realizar las mismas".
} 
| Revista: Ciencia, Tecnología y Política | Año 1 Número 1 | www.revistas.unlp.edu.ar/CTyP |

máticas estratégicas y en particular para este trabajo, fueron considerados aquellos SC relacionados a las geociencias, tanto desde el ámbito hidrocarburífero (AAPG), como de la minería (SEG). El principal objetivo de la AAPG es "apoyar las ciencias de la tierra" y a través de su Fundación otorga fondos para una gran variedad de programas educativos y de investigación que benefician a profesionales, estudiantes y público en general. Para cumplir este mandato brinda "financiamiento para becas a estudiantes de grado y postgrado de todo el mundo, para publicar en diferentes reuniones científicas, y cualquier otra actividad que permita promover la formación y mejorar el trayecto de los actuales y futuros profesionales". 3

En el caso de la SEG el objetivo es similar, orientado al "Avance en el conocimiento de la geología a través de la investigación científica de los yacimientos y recursos minerales, como su aplicación en la exploración, evaluación y extracción de los mismos"4. De esta manera busca fomentar la difusión y la producción científica básica y aplicada, a través de publicaciones, reuniones, simposios, conferencias, excursiones, cursos, etc. ${ }^{5}$

Ambas asociaciones se presentan como organizaciones profesionales sin fines de lucro, aunque cabe destacar que se encuentran patrocinadas y auspiciadas por compañías multinacionales de países centrales. Entre ellas se destacan: Shell, Chevron, Exxon Mobil, Apache, BP, Barrick Gold, Goldcorp, Hecla, Teck, entre otras decenas de empresas.

Los SC se conforman como la principal herramienta de inserción universitaria de estas asociaciones y sociedades. ${ }^{6}$ Los mismos se distribuyen por las universidades de cientos de países en todo el mundo y poseen miles de socios activos. En Argentina se destacan los SC de las Universidades Nacionales de La Plata, del Sur, Comahue, Río Negro, Salta y Universidad de Buenos Aires.

En cuanto a la organización interna de los SC, la figura de Advisor ocupa el vértice superior de su pirámide estructural y es indispensable para la conformación de la misma. Ser Advisor requiere formar parte de la academia mediante un cargo de profesor y paralelamente estar vinculado a la industria.? Los cargos restantes del comité ejecutivo, Presidente, Vice-Presidente, Secretario y Tesorero pueden

\footnotetext{
${ }^{3}$ Fragmento extraído y traducido de la página web de la AAPG: www.aapg.org (18/06/18)

${ }^{4}$ Fragmento extraído y traducido de la página web de la SEG: www.segweb.org (18/06/18)

5Sobre el reporte anual (2015) de la SEG Canada Foundation, una de las fundaciones que financian las actividades de la asociación, es interesante observar cuáles son las "investigaciones de vanguardia" que se buscan beneficiar, teniendo en cuenta la distribución económica según las diferentes commodities primarias de los distintos yacimientos estudiados. Prácticamente el $75 \%$ de los fondos se destinan a proyectos vinculados a la exploración y explotación de oro, cobre, zinc, níquel y metales especiales. ${ }^{6}$ Aunque también existen otros programas que buscan financiar actividades de jóvenes graduados. Entre ellos podemos destacar el apoyo económico a proyectos orientados a jóvenes investigadores (Student Research Grant), el programa para jóvenes graduados (Graduate Student Fellowship Program), ambos de la SEG. En el caso de la AAPG cuenta con una gran diversidad de becas para jóvenes estudiantes de postgrado. El requisito para su aplicación es ser miembro de estas asociaciones.

${ }^{7}$ En el caso de la SEG el profesor debe aplicar a una membresía especial (Fellow), la cual es evaluada por pares y depende del grado de reconocimiento personal del postulante. Es fundamental demostrar una vasta experiencia en el campo de la geología económica. En el caso del profesor, actual referente del SC de la SEG, pudo aplicar en 2008 luego de manifestar el hallazgo de un gran yacimiento mineral.
} 
ser ocupados por graduados/as y estudiantes. Los/las graduados/as son en general jóvenes profesionales que se encuentran realizando su tesis de doctorado. Participan en la organización interna, coordinando reuniones, actividades y administrando el cumplimiento burocrático que exige la organización. Finalmente, los/las estudiantes de grado son los miembros más numerosos y constituyen los principales destinatarios de los SC. ${ }^{8}$ Además de concurrir a reuniones y actividades, estos deben estar asociados a las organizaciones madre ${ }^{9}$ para acceder a cargos de gestión, o a diferentes beneficios como becas para congresos y cursos, acceso y descuento en publicaciones, fondos para realizar viajes al exterior o a diferentes yacimientos mineros de Argentina y Chile, entre otros. Por otro lado, participar en una sociedad internacional "permite ampliar los contactos profesionales, entre el sector privado, entes gubernamentales e instituciones académicas." ${ }^{\prime \prime}$

\section{Los Student Chapters en la FCNyM}

Actualmente, en la facultad funcionan activamente dos SC que dependen de la AAPG y la SEG. Por un lado, el primero fue propuesto desde la empresa, como bien fue expresado por su Advisor en la entrevista: "En 2010 se acercó gente cercana al gerente de Shell, con la propuesta de constituir el SC de la AAPG en la Facultad. En ese momento estaban muy interesados en expandir los SC al resto de Latinoamérica" (Advisor AAPG). Por otro lado, el SC de la SEG se fundó en 2003, aunque permaneció inactivo hasta el año 2008 cuando se retoma el proyecto a cargo de un nuevo Advisor académico.

Las actividades que desarrollan estos SC, son diversas y dependen tanto de la asociación fundadora como de la propia organización interna. Entre las actividades principales se encuentra la organización de charlas, conferencias y cursos de profesionales especializados en diversos temas, tanto del ámbito empresarial como científico-académico. Entre los cursos dictados se encuentra por ejemplo el dictado en noviembre de 2017 a cargo de una especialista brasileña, exclusivo para miembros del SC de la SEG, el cual trató sobre nociones básicas del programa de modelado geológico Leapfrog 3D"1. También se suelen organizar todos los años seminarios cortos (10hs) sobre "Depósitos epitermales: características, ejemplos y sus aplicaciones" y "Logueo de perforaciones en Minería". En 2013 se realizó el Workshop de la AAPG Argentina sobre Vaca Muerta, "Argentina's Vaca Muerta Draws", donde más de 50 profesionales de la industria intercambiaron opiniones sobre el potencial de explotación del recurso no convencional y su correlación con el boom del shale oil y shale gas de Estados Unidos. En mayo de 2015 se desarrolló el curso Geosciences Technology Workshop, "Extending Mature Fields' Life Cycles: the Role of New Technologies \& Integrated Strategies" organizado

\footnotetext{
${ }^{8} \mathrm{~A}$ partir de las entrevistas realizadas, se comprobó que tanto graduados/as como Advisors consideran que los/las principales beneficiados/as son los/las estudiantes, por diversas cuestiones. Por ejemplo tener contacto con profesionales que se desempeñan en otro ámbito, la transmisión de experiencias profesionales, temáticas particulares que no se abordan en las cursadas, y todo lo referido a la formación profesional de cada individuo a través de cursos, charlas, etc.

${ }^{9}$ En el caso de la AAPG la membresía de 10 USD anuales es financiada por Chevron.

${ }^{10}$ Ver: www.segweb.org (18/06/18)

${ }^{1}$ Se trata de un programa líder en el ámbito del modelado geológico de yacimientos minerales tanto desde el punto de vista de la exploración como explotación minera.
} 
por la AAPG en Capital Federal'12, como así también uno sobre sísmica en la FCNyM. Otro evento destacado, específicamente en el SC de la AAPG, es la participación en el "Imperial Barrel Award", una competencia anual donde los/las estudiantes resuelven problemas de la industria petrolera de manera creativa, en donde las empresas patrocinadoras de la asociación además de brindar una base de datos, designa los jurados del evento. Otra actividad relevante, en este caso desarrollada por la SEG, son viajes de campaña a los yacimientos minerales más importantes de la región.

Todas estas actividades están destinadas a una parte específica del estudiantado de la FCNyM y no a todo su espectro. Esto se ve claramente en el análisis de las encuestas realizadas, cuyos resultados arrojaron que de los 33 encuestados/as de 3er año solamente 2 habían escuchado hablar del tema. Por el contrario, en 4 to y 5 to año entre un 84 y 94\% de los/las encuestados/as tenían conocimiento de la existencia de los SC. Una tendencia similar se observa respecto al grado de participación de los/las estudiantes en los mismos, con 34 miembros activos entre ambos. De esta manera, queda explícito que el principal abordaje es sobre los/las estudiantes próximos/as a graduarse.

\section{Los Student Chapters y sus vínculos institucionales}

Autoridades de la Facultad aseguran que no existe un vínculo directo entre la institución con la AAPG y/o la SEG o sus SC: "la relación se genera directamente con los profesores referentes". No existe un espacio de discusión y evaluación que avale o no el funcionamiento de los SC y las actividades que en ellos se realizan. La institución no tiene injerencia sobre los contenidos temáticos o actividades que desarrollan, ya que son considerados por las propias autoridades "como cualquier otra actividad extracurricular que se realiza en la institución". Además, profesores Advisors y autoridades coincidieron en que la Universidad no obtiene ningún beneficio a través del desarrollo de sus actividades: "Es el primer vínculo entre la industria y el estudiante. Me parece que pasa por encima de la Universidad, no genera un beneficio directo a la institución, sino al alumno. Lo cual es lógico porque este es el objetivo, que se lleve un primer pantallazo del mundo profesional, yo invito profesionales, no académicos. Gerentes, gerentes de recursos humanos, gente que trabaja en responsabilidad social empresarial. Gente que le habla de lo mismo pero desde otro ámbito. Mostrar un poquito de cómo es la industria" (Advisor SEG). De la misma manera opinó la Advisor AAPG, "Los que se benefician realmente son los chicos."

De esta manera, el vínculo con la Universidad se genera a partir de que la misma brinda el espacio físico para desarrollar las actividades y de manera unilateral como institución apadrinadora. Aunque la institución no otorgue dinero, sí brinda legitimidad al conceder el aval institucional a la hora de presentar informes anuales y solicitar fondos para diferentes proyectos. "'La Facultad directamente funciona de by pass para que la industria llegue a los futuros profesionales" (Advisor SEG). Cabe destacar otros organismos que respaldan

\footnotetext{
${ }^{12}$ La inscripción fue abierta a estudiantes y profesionales con un costo de 250 USD, otorgando 3 becas de 150 USD para estudiantes miembros de la AAPG. Siendo estudiante avanzado de Buenos Aires o La Plata, la AAGGP (Asociación Argentina de Geólogos y Geofísicos Petroleros) otorga una ayuda económica adicional de 50 USD para la inscripción promocional y 50 USD extra para gastos de transporte, comida, etc.
} 
el funcionamiento del SC de la SEG como son el Instituto de Recursos Minerales (INREMI) dependiente de la Universidad Nacional de La Plata, la Dirección de Minería de la Provincia de Buenos Aires y, desde el año 2016, de la compañía Austral Gold Limited.13

Por otro lado, es evidente el interés de las corporaciones privadas que auspician a las asociaciones, de influir en la formación de los futuros profesionales: "Representan la primer apertura directa de la Universidad a las empresas"... "Las empresas les dan mucha importancia a los SC. Hay un fuerte respaldo de estas a solicitudes que vengan con el aval de la SEG" (Advisor SEG).

\section{Discusión y conclusiones}

De la investigación realizada en este trabajo sobre los SC, se puede observar que estas estructuras co-educacionales poseen similares formas de organización y funcionamiento entre sí. Con respecto a sus objetivos: "avanzar en el conocimiento de la ciencia geológica...", "mejorar la situación profesional de los geólogos vinculados a la temática"l4, entre otros, una mirada crítica de los mismos, nos permite suponer que parten de entender el avance científico como algo lineal que beneficiará al conjunto de la sociedad. Cabe repreguntarse algo ya planteado por Varsavsky (Varsavsky, 1969): “¿Por qué tanto interés norteamericano (...) en elevar nuestro nivel científico?". Si analizamos los objetivos en conjunto con las actividades desarrolladas por los SC y las temáticas abordadas, se observa que están íntimamente relacionadas a la exploración y explotación de recursos naturales estratégicos como hidrocarburos, metales preciosos y otros. Es evidente que estos fomentan la reproducción por parte de las universidades de los conceptos y procesos desarrollados en países centrales. Esto inevitablemente nos hará atender a las necesidades de las firmas multinacionales y no a las necesidades de nuestra región. Ante esta situación es importante tener presente el concepto del criterio de importancia desarrollado por Varsavsky para entender que es necesario pensar la ciencia y la educación desde una perspectiva de autonomía científica y soberanía nacional: "una teoría sobre petróleo no tiene el mismo interés en Suiza que en Venezuela” (Varsavsky, 1969). De esta manera se explicitan mecanismos que buscan homogeneizar nuestros procesos educativos y científicos con los provenientes de países centrales, fortaleciendo distintas formas de dependencia.

El ejemplo de la FCNyM, pone en evidencia el vínculo asimétrico que existe entre los SC, las instituciones públicas de enseñanza y las asociaciones estadounidenses (AAPG y SEG, en el caso estudiado). La noción que se genera de la empresa con respecto a la Universidad (en palabras de los miembros estudiantiles: "La empresa da prestigio a la Universidad") pone de manifiesto cómo los SC operan moldeando el sentido común de la comunidad académica, invadiéndolo de múltiples elementos de la lógica empresarial, mientras que se naturaliza el vínculo Universidad-empresa por parte de los Advisors y Autoridades. Se puede afirmar, entonces, como bien plantea Romero Rodríguez (2001) que: "En los planes de formación universitaria se registra una modificación en los fines académicos, que ya

\footnotetext{
${ }^{13}$ Ver: www.segweb.org/pdf/students/student-chapters/universidad-nacional-de-la-plata/Annual-Report.pdf (18/06/18)

14 Ver: www.foundation.aapg.org/programs (18/06/18)
} 
no responden sólo a la contribución del avance en el conocimiento, sino que se establecen en función de objetivos definidos por las necesidades de las empresas". Es dentro de esta cultura académica, inmersa en criterios empresariales, que se forman estudiantes aislados de una mirada crítica capaz de cuestionar las lógicas impuestas y que termina dando forma al perfil profesional del geólogo actual.

En este sentido es fundamental que nos planteemos qué proyecto nacional queremos, y cómo hacer que éste cimente las bases para un desarrollo soberano, inclusivo y equitativo real, donde no se busque la sinergia entre las partes del sistema con la ganancia económica como precepto, sino que su objetivo sea la resolución de los problemas de nuestro pueblo, como dice Varsavsky (1969): "Un país es su pueblo -pasado, presente y futuro- y toda decisión debe comenzar por allí, por su existencia y por sus necesidades".

En la FCNyM, las Asociaciones y Sociedades que desarrollan los SC, aprovechan la expectativa de muchos estudiantes, urgidos por la necesidad de conseguir trabajos bien remunerados y que se supone otorgarán prestigio y privilegios sociales, para desarrollar actividades que en función de estos objetivos pueden ir en contra de los intereses y necesidades de la Universidad Pública y del país. No solo formando sutilmente profesionales cada vez más tecnócratas, sino profundizando las lógicas culturales que permiten que estos sistemas sigan perpetuándose en los diferentes ámbitos, ya sea dentro de la Universidad, la sociedad, el sector productivo, científico o tecnológico.

Para finalizar, cabe aclarar que no es intención de este trabajo plantear el rechazo total de los vínculos entre la universidad y las empresas. Al contrario, consideramos necesario el encuentro entre el sector productivo y la Universidad como actores fundamentales dentro de un proyecto nacional soberano. Sin embargo, entendemos que desde la Universidad pública se deben dejar de inculcar los principios impuestos por los países dominantes, y en su lugar, dar espacio a la reflexión sobre el modelo científico tecnológico que necesitamos, lo cual implicaría un paso muy importante en el proceso de descolonización cultural. En este marco queremos finalizar este trabajo con una propuesta: crear Capítulos Estudiantiles que promuevan actividades atendiendo a las necesidades particulares de nuestra región. Que estos capítulos interactúen con empresas nacionales, organismos del Estado, organizaciones comunitarias, municipios y otros actores de la comunidad, como así también con instituciones científico-tecnológicas públicas. Proponemos además que estos capítulos se constituyan con el aval de los Centros de Estudiantes de las facultades donde se desarrollen y de otros espacios institucionales de la universidad, situación que en la actualidad no ocurre.

\section{Bibliografía}

A Nairdorf, J. (2005). Privatización del Conocimiento Público en Universidades Públicas. Espacio público y privatización del conocimiento. Estudios sobre políticas universitarias en América Latina. p. 101 - 161. Buenos Aires, Argentina.

Rodriguez, R. (2001). Globalización, Mercado Mundial y Nuevos Escenarios para la Educación Superior. Memorias del Congreso Convergente IESM/IESLA. Veracruz, México.

Varsavsky, O. (1969). Ciencia, Política y Cientificismo. Ediciones de la Feria/CEAL. Buenos Aires, Argentina. 